\title{
ADAPTIVE GAIT PARAMETERS ADJUSTMENT STRATEGY FOR A HEXAPOD ROBOT WALKING ON STAIRS BASED ON 3D TERRAIN PERCEPTION
}

\author{
YUE ZHAO and FENG GAO* \\ State Key Laboratory of Mechanical System and Vibration, Shanghai Jiao Tong University, \\ Shanghai, 200240, China \\ E-mail:yue.zhao@sjtu.edu.cn,fengg@sjtu.edu.cn \\ www.sjtu.edu.cn \\ YUAN TIAN and LIHENG MAO \\ State Key Laboratory of Mechanical System and Vibration, Shanghai Jiao Tong University, \\ Shanghai, 200240, China \\ E-mail: tianyuan_123abc@sjtu.edu.cn,mr.liheng@sjtu.edu.cn
}

\begin{abstract}
Legged robots have advanced potential to accomplish detecting, carrying, operating and rescuing tasks instead of human beings in complex environments. In real applications, walking over and climbing the obstacles is a more urgent issue to be solved for legged robots comparing with bypassing obstacles. However, few papers involve approaches of climbing obstacles for legged robots. A perception-based gait parameters adjustment method for a hexapod robot walking on stairs is presented in this paper. To fulfil requirements of walking stability and kinematic feasibility, this method takes advantage of the superior mobility of the six-legged robot and adapts the gait parameters autonomously based on the terrain perception. First, a robot state estimation method is proposed, which figures out position and posture of the robot. Then, a perceptionbased robot-stairs interference model is proposed, which describes the relationship between the robot and stairs. After that, a method to calculate appropriate gait parameters to climb stairs is developed. Finally, the scheme is integrated on the hexapod robot Qingzhui and tested by real experiments.
\end{abstract}

\section{Introduction}

Legged robots have advanced potential to help people in daily life compared with wheeled robots and tracked robots $[1 ; 2]$. One major reason is that there are many steps or stairs in people's life, which are challenging for wheeled and tracked robots. Although legged robots have the ability to overcome these complex environments, the walking velocity is an area that needs improvement. Traditional climbing stairs methods rely on offline gait planning or specific online gait planning which is exclusively used for steps or stairs. With these methods, robots may lack autonomy and rapidity. Therefore, an adaptive gait parameters adjustment strategy for a hexapod robot walking on stairs based on 3D terrain perception is proposed. The robot can accomplish climbing stairs rapidly without collision by autonomously adjusting parameters of the regular dynamic alternating tripod walking gait.

With respect to the issue of gait planning, many researchers have performed much work on legged robots. Marc Raibert et al. [3] proposed a gait coordination algorithm which was utilized on BigDog to walk on inclined and rough terrain. Nathan Ratliff et al. [4] presented a novel method CHOMP for continuous path refinement and demonstrated it with a walking quadruped robot. Dominik Belter et al. [5; 6] presented a method for real-time motion planning on a rugged terrain and it was validated with a hexapod robot. Michael P Murphy et al. [7] introduced a compact quadruped robot designed to support the research applications. Alexander Shkolnik et al. [8] proposed a motion planning algorithm for bounding over rough terrain developed by 
Rapidly Exploring Random Trees (RRTs). Yuan Tian and Feng Gao [9-11] decomposed the motion planning on irregular terrain into two main steps: the foothold selection and whole body configuration planner. Marco Hutter et al. [12-14] split the locomotion control of ANYmal into five key components: state estimation, localization and mapping, navigation, foothold and motion planning, as well as whole body control. Gerardo Bledt et al. [15-18] used an eventbased finite state machine to define the Cheetah's gait. Marko Bjelonic et al. [19] introduced a six-legged robot which used stereo vision and proprioceptive sensing based terrain perception for adaptive control. Although many researchers have performed much work on gait planning, there is still need to develop an adaptive gait parameters adjustment strategy for a hexapod robot walking on stairs based on 3D terrain perception.

The reminder of this paper is organized as follows. Section 2 introduces the robot system briefly. Section 3 presents the adaptive gait parameters adjustment strategy in details. Section 4 discusses the experiments that were used to validate the effectiveness of our method. Finally, Section 5 concludes the paper.

\section{Robot System Overview}

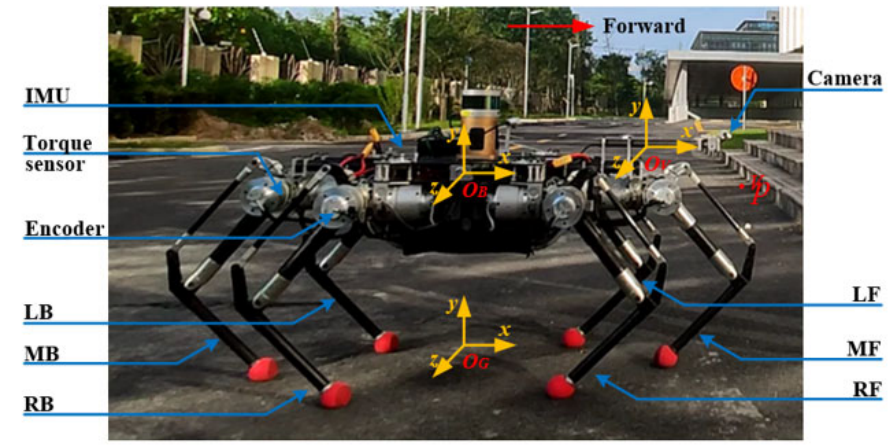

(a)

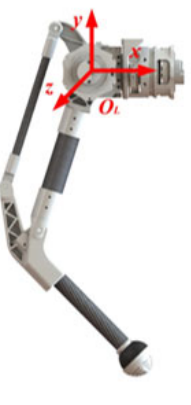

(b)

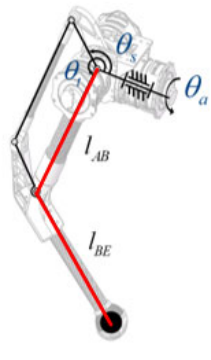

(c)

Figure 1. Robot system overview. (a) The photograph of the hexapod robot Qingzhui (b) Leg structure and definition of hip coordinate system (c) Mechanism schematics of the leg

This paper proposes a perception-based stair-climbing planning method for a prototypic sixlegged walking robot Qingzhui. As shown in Figure 1(a), Qingzhui hosts one IMU, eighteen encoders and eighteen torque sensors as proprioceptive sensors and one RGB-D camera as perceptive sensor. In our earlier work [9], a force-based online gait planning is applied in the hexapod robot. The motion control loop includes torque sensory feedback, IMU data feedback, the gait control and the leg kinematics. The motion control frequency is about $1000 \mathrm{~Hz}$. Based on this motion plan, the hexapod robot can carry a 20 kilogram package, walk on a slope with 30 degrees, step over $25.0 \mathrm{~cm}$ ditch and can walk at $0.6 \mathrm{~m} / \mathrm{s}$ which is similar to the normal walk velocity of human beings.

As shown in Figure 1(b), the leg has three degrees of freedom, corresponding to the hip abduction-adduction degree, rotation degree of the thigh and rotation degree of the shank. With the use of proprioceptive sensors, the position and force of each tip can be calculated in every millisecond. The position of toe can be written as follows:

$$
\begin{gathered}
x=l_{A B} \cos \theta_{t}+l_{B E} \cos \theta_{s} \\
y=\left(l_{A B} \sin \theta_{t}+l_{B E} \sin \theta_{s}\right) \cos \theta_{a}
\end{gathered}
$$




$$
z=\left(l_{A B} \sin \theta_{t}+l_{B E} \sin \theta_{s}\right) \sin \theta_{a}
$$

where $l_{A B}$ is the length of the thigh, $l_{B E}$ is the length of the shank, $\theta_{t}$ is the rotation degree of the thigh, $\theta_{s}$ is the rotation degree of the shank, and $\theta_{a}$ is the hip abduction-adduction degree. These parameters are illustrated in Figure 1(c).

The force of toe can be calculated as follows:

$$
\begin{gathered}
J=\left[\begin{array}{ccc}
-l_{A B} \sin \theta_{t} & -l_{B E} \sin \theta_{s} & 0 \\
l_{A B} \cos \theta_{t} \cos \theta_{a} & l_{B E} \cos \theta_{s} \cos \theta_{a} & -\left(l_{A B} \sin \theta_{t}+l_{B E} \sin \theta_{s}\right) \sin \theta_{a} \\
l_{A B} \cos \theta_{t} \sin \theta_{a} & l_{B E} \cos \theta_{s} \sin \theta_{a} & \left(l_{A B} \sin \theta_{t}+l_{B E} \sin \theta_{s}\right) \cos \theta_{a}
\end{array}\right] \\
F_{t i p}=\left[\begin{array}{l}
F_{t x} \\
F_{t y} \\
F_{t z}
\end{array}\right]=\left(J^{T}\right)^{-1}\left[\begin{array}{c}
\tau_{s} \\
\tau_{t} \\
\tau_{a}
\end{array}\right]
\end{gathered}
$$

where $J$ is the Jacobi matrix, $F_{\text {tip }}$ is the force of the toe, and $\tau$ is the joint torque which can be captured from the torque sensor mounted on the joint.

\section{Method}

The whole method is based on the superior mobility of the six-legged robot Qingzhui. Qingzhui is applied with the dynamic alternating tripod walking gait which can accomplish most terrain traversing tasks. However, stairs is a non-contiguous space, which makes it difficult for the robot to adjust gait parameters only based on the current robot state. Therefore, visual feedforward is necessary while climbing stairs. As shown in Figure 2, the whole method can be divided into three parts. First, a robot state estimation method based on the specific walking gait is proposed. Then, a stairs modeling method based on the visual perception is proposed. Finally, an online parameter generation method with respect to the leg interference problem is proposed.

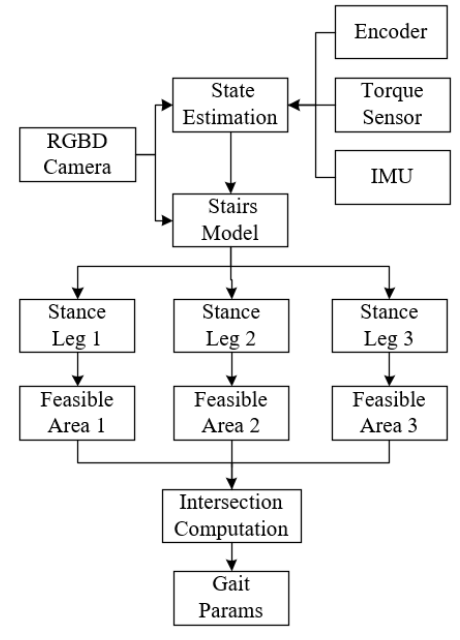

Figure 2. The control architecture of calculating

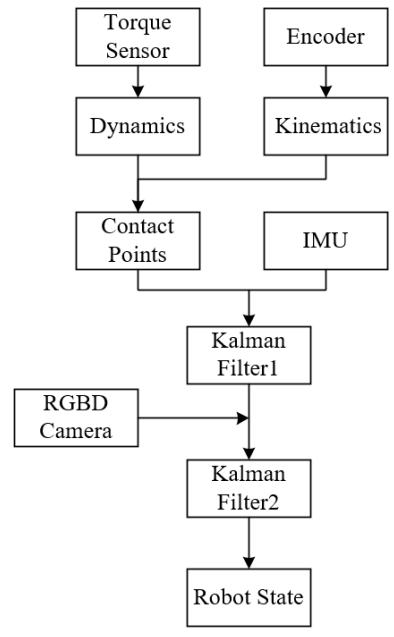

Figure 3. The flow chart of the robot state estimation 


\subsection{Robot state estimation}

To climb the stairs, the first question to be solved is how to describe the relationship between the robot and the stairs. As shown in Figure 3, a robot state estimation method is proposed in this paper. First, a state estimation method based on the robot kinematics is utilized to get the robot's positions and RPY (roll, pitch, and yaw) angles. The first Kalman filter takes into account the proprioceptive sensors. Meanwhile, the ICP (Iterative Closest Point) method is utilized to calculate the position and posture transformation matrix between every two point clouds of the stairs which are captured by the RGB-D camera. A relative relationship between the robot and the stairs can be identified by iterative correction with the second Kalman filter.

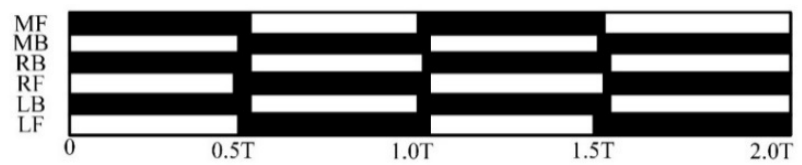

Figure 4. Diagram of gait sequence. Feet in support phase are filled with black and feet in swing are filled with white.

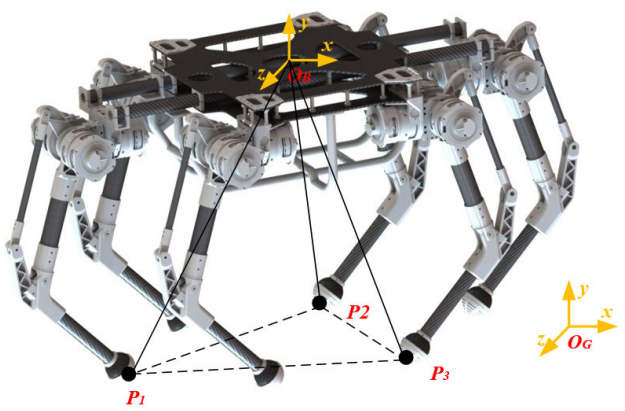

Figure 5. Definitions of the feet in stance phase

The robot uses the same walking gait when it walks on the even terrain and climbs stairs. The gait sequence is shown in Figure 4. The gait guarantees that at least three feet are supporting the whole body to prevent the robot falling over. Middle front (MF) leg, right back (RB) leg, and left back (LB) leg move as group A, while middle back (MB) leg, right front (RF) leg, and left front (LF) leg move as group B. Each foot force can be calculated based on the torque sensors. If the sum of support force of group A is larger than group B, it is believed that group $\mathrm{A}$ is in support phase and vice versa. Based on this, the positon and posture of the robot can be calculated trough forward kinematics. Assuming that group A is in stance phase, each foot point position can be expressed in both body coordinate system and global coordinate system. The position and posture transformation matrix can be calculated by the geometrical constraint. As shown in Figure 5, three contact feet are expressed as $P_{1}, P_{2}$, and $P_{3}$. The length between body center and the contact feet is same in body coordinate and global coordinate. The global robot center position and the rotation matrix can be calculated as follows:

$$
\begin{gathered}
\left(\begin{array}{c}
{ }^{\mathrm{B}} P_{i}^{\mathrm{st}} \\
1
\end{array}\right)={ }_{i \mathrm{~L}}^{\mathrm{B}} \boldsymbol{T}\left(\begin{array}{c}
{ }^{\mathrm{L}} P_{i}^{\mathrm{st}} \\
1
\end{array}\right), i=1,2,3 \\
\left\|{ }^{\mathrm{G}} P_{i}^{\mathrm{st}}-{ }^{\mathrm{G}} O_{\mathrm{B}}\right\|_{2}=\left\|{ }^{\mathrm{B}} P_{i}^{\mathrm{st}}\right\|_{2}, i=1,2,3 \\
{ }^{\mathrm{G}} P_{i}^{\mathrm{st}}-{ }^{\mathrm{G}} O_{\mathrm{B}}={ }_{\mathrm{B}}^{\mathrm{G}} R^{\mathrm{B}} P_{i}^{\mathrm{st}}, i=1,2,3
\end{gathered}
$$




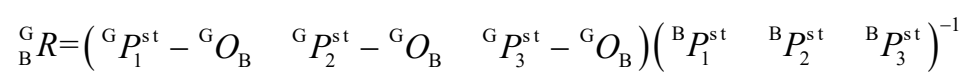

After that, the kinematics estimation result is integrated with the IMU data and ICP result, and the final robot state is confirmed.

\subsection{Stairs modeling method}

Since the position and posture of the robot has been calculated, each point cloud acquired from the RGB-D camera can be transferred into the global coordinate system. As shown in Figure 1(a), the point obtained from the camera can be transferred into the global coordinate system as follows:

$$
{ }^{G} p={ }_{B}^{G} T \square_{V}^{B} T \square^{V} p
$$

where point ${ }^{V} p$ with respect to vision coordinate system is obtained from the RGB-D camera, ${ }_{V}^{B} T$ is the identification matrix between the vision coordinate system and the body coordinate system, ${ }_{\mathrm{B}}^{\mathrm{G}} T$ is the transportation matrix between the body coordinate system and global coordinate system, and ${ }^{G} p$ is the point with respect to global coordinate system.

As shown in Figure 6, there are some artifacts which are caused by the chattering phenomena and the erroneous measurement appearing on the edges of stairs. To solve this problem, the horizontal planes are estimated by RANSAC (Random Sample Consensus) method from the point cloud and center area is kept by ignoring 5\% points near the edge. An ICP method is used to stitch the stairs. The earlier point cloud is the source, and the later point cloud is the target. While stitching the stairs, the result can also be used to correct the position and posture of the robot.

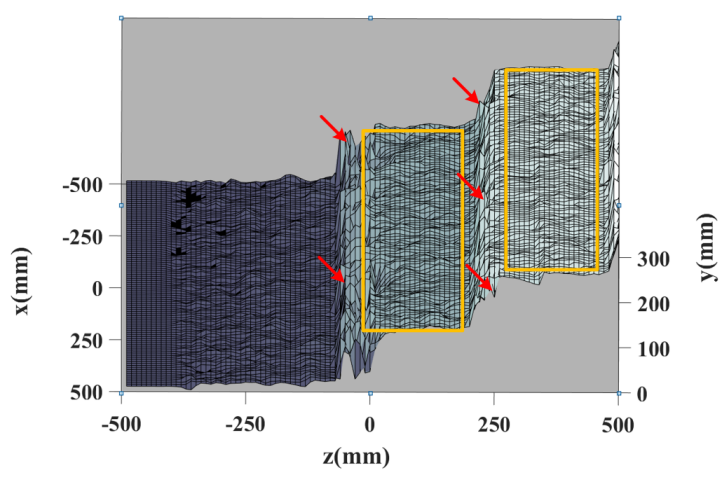

Figure 6. Stairs map captured by the RGB-D camera (red arrows point to some artifacts and the yellow rectangles represent the estimated planes)

Up to this point, both the robot and the stairs can be expressed in global coordinate system, and the perception-based robot-stairs interference model can be built. Based on this, the relative position and posture between the robot and the stairs can be utilized in next algorithm.

\subsection{The calculating method of gait parameters}

The calculating method of gait parameters aims at finding appropriate step length and step height for the robot to walk on stairs both stably and rapidly. For each foot, there is an area along the motion direction. It may contain one step, two steps, three steps and even more. However, feasible region is not only decided by the plane but also the interference relationship between the robot and the stairs. 
The hexapod robot Qingzhui has two types of knee joints. For each plane of the stairs, it can be divided into three areas: far area, middle area and near area. The default length of near area and far area is $5 \mathrm{~cm}$, while it can be changed by the interference model between legs and stairs.

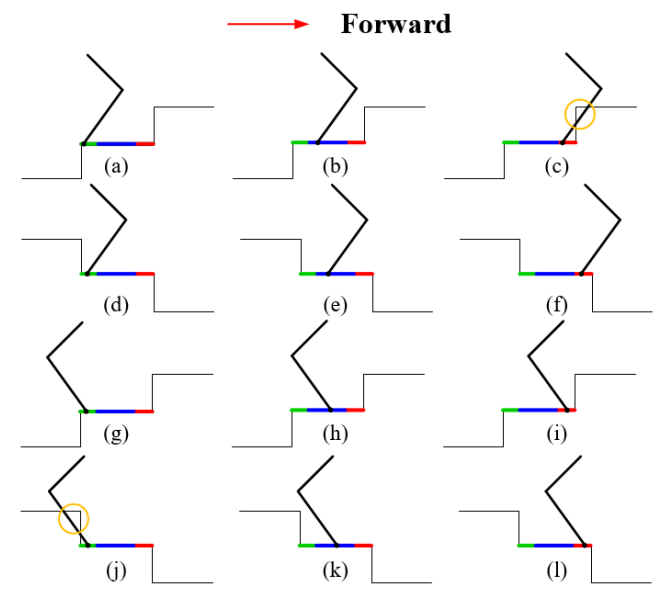

Figure 7. The relationship between stairs and leg. (a-c) Climb up stairs with type-a leg (d-f) Walk down stairs with type-a leg (g-i) Climb up stairs with type-b leg (j-1) Walk down stairs with typeb leg

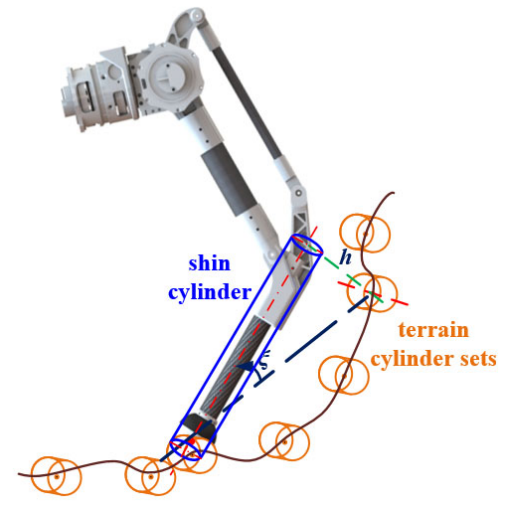

Figure 8. Leg collision interference model

As shown in Figure 7, the leg may collide the stairs, and the collision often happens between the shin and the stairs edges. As shown in Figure 8, the shin can be expressed as a cylinder and the stairs can be expressed as many cylinders. The distance between axes can be one condition of collision and it can be calculated as follows:

$$
\begin{gathered}
d_{i}=\frac{\left|\left(\overrightarrow{s_{l}} \times \overrightarrow{s_{i}}\right) \square \overrightarrow{O P}\right|}{\left|\overrightarrow{s_{l}} \times \overrightarrow{s_{i}}\right|}, i=1,2,3, \square, N \\
s a_{i}=\left\{\begin{array}{ll}
1, \text { if } d_{i}<\left(r_{l}+r_{i}\right) \\
0,
\end{array}, i=1,2,3, \square \square, N\right.
\end{gathered}
$$

where $\overrightarrow{s_{l}}$ is the axis of the shin cylinder, $\overrightarrow{s_{i}}$ is the $i_{t h}$ terrain cylinder, $r_{l}$ is the radius of the shin cylinder and $r_{i}$ is the radius of the $i_{t h}$ terrain cylinder. Once $s a_{i}$ equals one, the collision will happen and this parameter needs to be excluded.

After that, each support foot has a feasible area, and it can be transferred to feasible sections of step length and step height. By using intersection computation of three feasible sections of the support feet, the gait parameters in next step can be confirmed.

\section{Experiments}

This paper proposes a perception-based stair-climbing method by adapting gait parameters autonomously for the hexapod robot Qingzhui. To verify the proposed method, experiments were carried out on the robot. The robot walked up and down the three $130 \times 300 \mathrm{~mm}$ stairs autonomously without the detailed parameters of the environment. By adapting the step length and step height, the walking gait took the robot from level ground up the stairs to level ground and from level ground down the stairs to level ground. 
Figure 9(a) shows the image flows of the robot walking on the stairs. Figure 9(b) shows the pitch angle of the robot. The robot climbed up the stairs first, and then walked down the stairs. Trajectories of the robot center point and each tip are shown in Figure 9(c). The robot body center trajectory is illustrated as black points, and six feet trajectories are illustrated as colorful points. The foothold sections were appropriate according to the figure. The feet position and force in hip coordinate system are shown in Figure 10. The position trajectories of symmetrical legs were approximately symmetrical and no saltation were found in $\mathrm{x}$ and $\mathrm{z}$ directions. The $\mathrm{x}$ direction force is small, and there is no too large force along $\mathrm{z}$ direction. These validate no collisions occur while the robot walking on stairs.

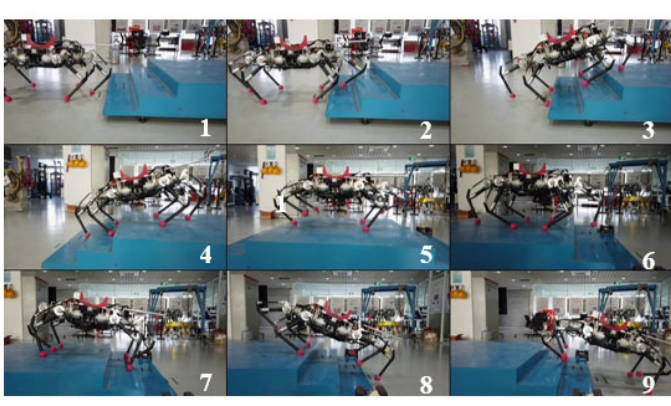

(a)

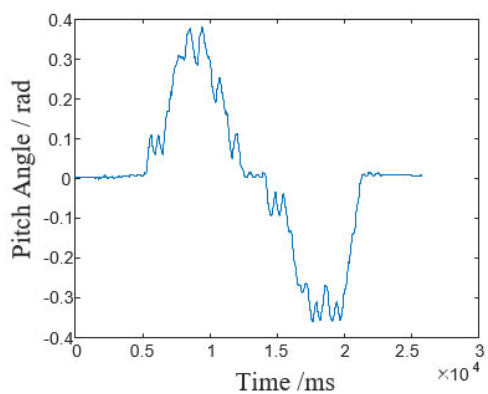

(b)

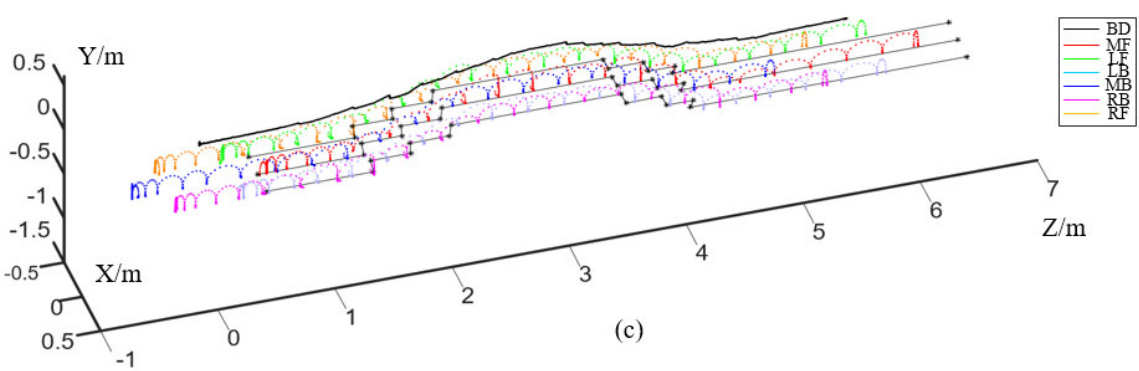

Figure 9. Experiment results. (a) Image flows of the robot climbing the stairs (b) Change of pitch angle when robot climbing the stairs (c) The trajectory of the robot center point and six feet

(a)

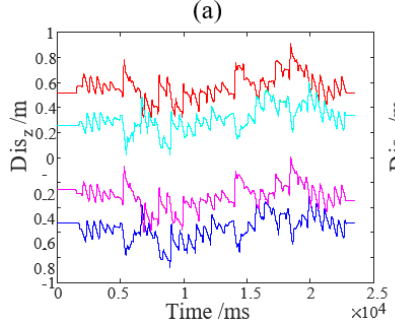

(d)

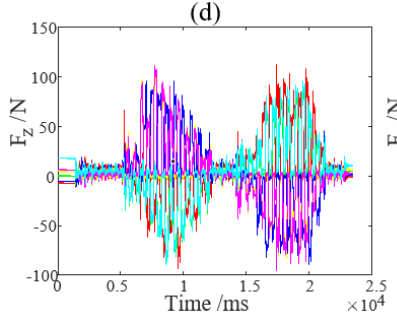

(b)

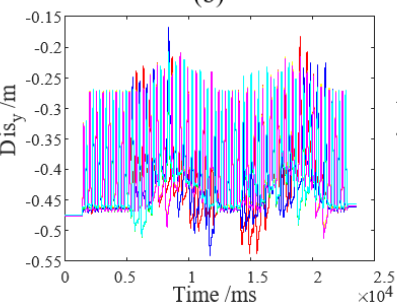

(e)

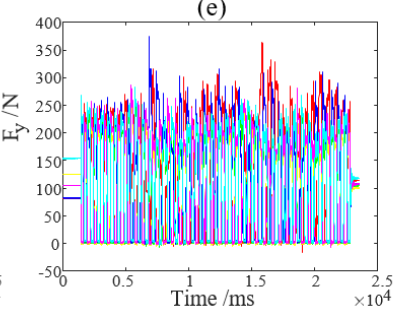

(c)

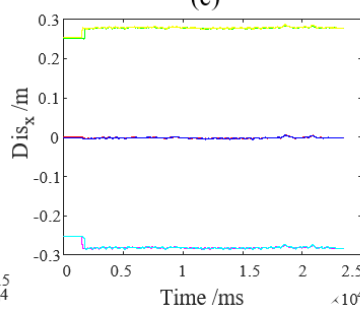

(f)

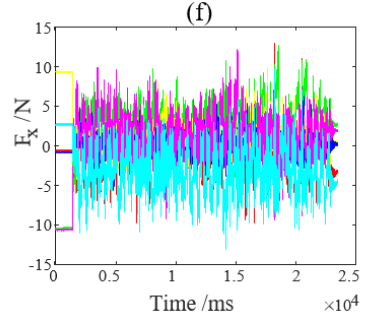

Figure 10. The force and position of each foot. (a) z-direction movement (b) y-direction movement (c) x-direction movement (d) z-direction force (e) y-direction force (f) $x$-direction force 


\section{Conclusion}

In this paper, we proposed an adaptive gait parameters adjustment strategy for a hexapod robot Qingzhui walking on stairs based on 3D terrain perception. To the best of the authors' knowledge, seldom studies have worked on the related issues. To accomplish climbing stairs, most online researches need to plan the robot and feet trajectory step by step while climbing, which takes a lot of time in computation. Our method does not change the motion planning, but changes the gait parameters include step length and step height, which can be finished in almost $100 \mathrm{~ms}$. Meanwhile, we plan the motion two steps ahead, and the motion is smoother.

\section{Acknowledgments}

This work was supported by the National Nature Science Foundation of China (Grant No. U1613208 and Grant No. 51927809), the National Key Research and Development Plan of China (Grant No. 2017YFE0112200), and the European Union's Horizon 2020 research and innovation programme under the Marie Skłodowska-Curie grant (Grant No. 734575).

\section{References}

1. M. Wermelinger, P. Fankhauser, R. Diethelm, P. Krüsi, R. Siegwart and M. Hutter. 2016 IEEE/RSJ International Conference on Intelligent Robots and Systems (IROS). 1184(2016).

2. P.-B. Wieber, R. Tedrake and S. Kuindersma, Springer handbook of roboticsr.: 1203(2016).

3. M. Raibert, K. Blankespoor, G. Nelson and R. Playter. Ifac Proceedings Volumes. 41, 10822(2008).

4. N. Ratliff, M. Zucker, J.A. Bagnell and S. Srinivasa. IEEE International Conference on Robotics \& Automation. (2009).

5. D. Belter and P. Skrzypczynski. Journal of Field Robotics. 28, 497(2011).

6. D. Belter and P. Skrzypczyński. IFAC Proceedings Volumes. 44, 6918(2011).

7. M.P. Murphy, A. Saunders, C. Moreira, A.A. Rizzi and M. Raibert. The International Journal of Robotics Research. 30, 145(2011).

8. A. Shkolnik, M. Levashov, I.R. Manchester and R. Tedrake. The International Journal of Robotics Research. 30, 192(2011).

9. Y. Tian and F. Gao. Robotica. 36, 333(2018).

10. Y. Tian, F. Gao, J. Liu and X. Chen. Science China Technological Sciences. 62, 597(2019).

11. Q. Sun, F. Gao and X. Chen. Robotica. 36, 1048(2018).

12. M. Hutter, C. Gehring, D. Jud, A. Lauber, C.D. Bellicoso, V. Tsounis, J. Hwangbo, K. Bodie, P. Fankhauser and M. Bloesch. 2016 IEEE/RSJ International Conference on Intelligent Robots and Systems (IROS). 38(2016).

13. M. Hutter, C. Gehring, A. Lauber, F. Gunther, C.D. Bellicoso, V. Tsounis, P. Fankhauser, R. Diethelm, S. Bachmann and M. Blösch. Advanced Robotics. 31, 918(2017).

14. P. Fankhauser and M. Hutter. Research Features. 54(2018).

15. G. Bledt, M.J. Powell, B. Katz, J. Di Carlo, P.M. Wensing and S. Kim. 2018 IEEE/RSJ International Conference on Intelligent Robots and Systems (IROS). 2245(2018).

16. J. Di Carlo, P.M. Wensing, B. Katz, G. Bledt and S. Kim. 2018 IEEE/RSJ International Conference on Intelligent Robots and Systems (IROS). 1(2018).

17. Q. Nguyen, M.J. Powell, B. Katz, J. Di Carlo and S. Kim. 2019 International Conference on Robotics and Automation (ICRA). 7448(2019).

18. B. Katz, J. Di Carlo and S. Kim. 2019 International Conference on Robotics and Automation (ICRA). 6295(2019).

19. M. Bjelonic, N. Kottege, T. Homberger, P. Borges, P. Beckerle and M. Chli. Journal of Field Robotics. 35, 1063(2018). 